\title{
Modeling Bounded Rationality in Capacity Allocation Games with the Quantal Response Equilibrium
}

Yefen Chen

Xuanming Su

University of Pennsylvania

Xiaobo Zhao

Follow this and additional works at: https://repository.upenn.edu/fnce_papers

Part of the Finance and Financial Management Commons, and the Operations and Supply Chain Management Commons

\section{Recommended Citation}

Chen, Y., Su, X., \& Zhao, X. (2012). Modeling Bounded Rationality in Capacity Allocation Games with the Quantal Response Equilibrium. Management Science, 58 (10), 1952-1962. http://dx.doi.org/10.1287/ mnsc. 1120.1531

This paper is posted at ScholarlyCommons. https://repository.upenn.edu/fnce_papers/115

For more information, please contact repository@pobox.upenn.edu. 


\title{
Modeling Bounded Rationality in Capacity Allocation Games with the Quantal Response Equilibrium
}

\author{
Abstract \\ We consider a supply chain with a single supplier and two retailers. The retailers choose their orders \\ strategically, and if their orders exceed the supplier's capacity, quantities are allocated proportionally to \\ the orders. We experimentally study the capacity allocation game using subjects motivated by financial \\ incentives. We find that the Nash equilibrium, which assumes that players are perfectly rational, \\ substantially exaggerates retailers' tendency to strategically order more than they need. We propose a \\ model of bounded rationality based on the quantal response equilibrium, in which players are not perfect \\ optimizers and they face uncertainty in their opponents' actions. We structurally estimate model \\ parameters using the maximum-likelihood method. Our results confirm that retailers exhibit bounded \\ rationality, become more rational through repeated game play, but may not converge to perfect rationality \\ as assumed by the Nash equilibrium. Finally, we consider several alternative behavioral theories and show \\ that they do not explain our experimental data as well as our bounded rationality model.
}

\section{Keywords}

bounded rationality, capacity allocation, supply chain, quantal response equilibrium, Nash equilibrium

\section{Disciplines}

Finance and Financial Management | Operations and Supply Chain Management 


\title{
Modeling Bounded Rationality in Capacity Allocation Games with the Quantal Response Equilibrium
}

\author{
Yefen Chen*, Xuanming $\mathrm{Su}^{\dagger}$, Xiaobo Zhao* \\ Submitted April 2011. Revised October 2011.
}

\begin{abstract}
:
We consider a supply chain with a single supplier and two retailers. The retailers choose their orders strategically and if their orders exceed the supplier's capacity, quantities are allocated proportionally to the orders. We experimentally study the capacity allocation game using subjects motivated by financial incentives. We find that the Nash Equilibrium, which assumes that players are perfectly rational, substantially exaggerates retailers' tendency to strategically order more than they need.

We propose a model of bounded rationality based on the Quantal Response Equilibrium, in which players are not perfect optimizers and they face uncertainty in their opponents' actions. We structurally estimate model parameters using the maximum likelihood method. Our results confirm that retailers exhibit bounded rationality, become more rational through repeated game play, but may not converge to perfect rationality as assumed by the Nash equilibrium. Finally, we consider several alternative behavioral theories and show that they do not explain our experimental data as well as our bounded rationality model.
\end{abstract}

Keywords: bounded rationality; capacity allocation; supply chain; quantal response equilibrium; Nash equilibrium.

\section{Introduction}

Capacity allocation is an important issue in supply chain management. When demand is high, there may be a capacity shortfall in the supply chain. Since it is often infeasible to expand capacity in the short term, a capacity-constrained supplier has to divide the limited supply among prospective retailers. Consequently, retailers may choose their orders strategically, and such behavior has profound implications on profits and supply chain efficiency. In this paper, we conduct laboratory experiments to study retailers' ordering behavior in capacity allocation games. We identify

*Department of Industrial Engineering, Tsinghua University

${ }^{\dagger}$ Haas School of Business, University of California, Berkeley 
systematic deviations from standard game-theoretic predictions, and develop a model of bounded rationality to explain the empirical regularities in our data.

Capacity allocation games have been studied extensively in the literature. The game-theoretic framework is perhaps the most popular method in analyzing how different allocation mechanisms affect retailers' ordering behavior and supply chain performance. Cachon and Lariviere (1999a, 1999b) investigated three allocation schemes: proportional, linear and uniform. The uniform allocation rule is "truth-inducing," while the proportional and linear allocation rules are shown to be "order-inflating," i.e., they may induce retailers to order more than they need so as to secure higher allocated quantities; such distorted information may cause the bullwhip effect (Lee et al. 1997). Another mechanism that has been proposed is the turn-and-earn system (Cachon and Lariviere 1999c), in which capacity is allocated based on past sales. There is also a large stream of existing work that examines the role of pricing mechanisms in allocating scarce capacity (e.g., Dewan and Mendelson 1990). All the papers above characterize the retailers' behavior using the classic concept of Nash equilibrium.

Although the Nash equilibrium provides a reasonable and valuable method of analysis, it is based on two strong assumptions. First, every player is a perfect optimizer who always takes an action to maximize her expected payoff. Second, every player has perfect knowledge of opponents' decision models and thus can perfectly infer what courses of actions her opponents will choose. In capacity allocation games, both assumptions may not hold. In this paper, we consider bounded rationality in capacity allocation games using the notion of quantal choice (Luce, 1959): all possible choice alternatives are candidates for selection, but more attractive alternatives (yielding higher utility) are chosen with larger probability. In a game-theoretic setting, such noisy optimization has two implications. First, players are no longer able to always optimize individual payoffs. Second, since their opponents also exhibit noisy decision-making, players face strategic uncertainty, i.e., they are not sure what their opponents will do. These two features allow us to relax the restrictive assumptions described above.

Experimental studies of rationality in game theory include McKelvey and Palfrey (1992), who find that subjects frequently fail to choose the unique Nash equilibrium actions, although they are able to make fewer errors over time with learning. McKelvey and Palfrey (1995) suggest that such errors may be due to bounded rationality and that these errors can be modeled by using latent utility components that are not reflected in pecuniary payoffs. They introduce the Quantal Response Equilibrium (QRE) framework, in which all players select actions according to the quantal choice model. Many general properties (e.g., existence, uniqueness, comparative statics) have been 
developed for models with both discrete and continue action sets (Chen et al. 1997, Anderson et al. 2002). The QRE framework has reasonable good predictive power in many game-theoretic settings (see Goeree and Holt 2001), including alternating-offer bargaining (Goeree and Holt 2000), coordination games (Anderson et al. 2001), auctions (Anderson et al. 1998, Goeree et al. 2002), constant-sum centipede games (Fey et al. 1996), traveler's dilemma games (Capra et al. 1999), and pricing contracts (Lim and Ho 2007, Ho and Zhang 2008). Although the QRE framework is wellestablished in behavioral economics, it is relatively new in the operations management literature; e.g., Su (2008) studies single-player newsvendor decisions using the quantal choice framework. We wish to begin studying multi-player applications of the QRE framework by first considering a simple capacity allocation game.

In this paper, we consider a supply chain with one supplier and two retailers. We conduct laboratory experiments to study retailers' ordering behavior in capacity allocation games. The experiment is designed as a one-shot game and subjects receive cash payments based on their performance in the experiments. We focus on the proportional allocation rule: retailers receive allocated quantities that are proportional to their orders, so they have an incentive to inflate their orders so as to secure more favorable allocated quantities when facing capacity shortfalls. The experimental data suggest that the subjects do not make decisions as predicted by the Nash equilibrium. To explain the experimental observations, we consider models of bounded rationality based on the QRE concept. We structurally estimate the parameters of the models and show that our models fit the data reasonably well. Our results show that the QRE can be a useful tool for analyzing strategic interactions in operational settings.

In the next section, we describe our capacity allocation model and present a standard gametheoretic analysis of the model. In Section 3, we describe the experimental design and provide some general statistical results. In Section 4, we develop models of bounded rationality and use them to analyze the capacity allocation games. In Section 5, we structurally estimate parameters of the models using maximum likelihood. In section 6, we consider several alternative behavioral theories and show that they do not explain the experimental data as well as our bounded rationality models. Finally, we present concluding remarks in Section 7.

\section{Capacity Allocation Game}

In this section, we introduce the capacity allocation game in a supply chain consisting of one supplier and two retailers. The supplier has a limited capacity $K$. Each retailer $i$ submits an order $x_{i}$ to the supplier. We use $x_{-i}$ to denote the order of retailer $i$ 's competitor. Both orders will be 
filled if the supplier has sufficient capacity, i.e., $K \geq x_{i}+x_{-i}$. Otherwise, the supplier divides its capacity among the two retailers using the proportional allocation scheme: each retailer $i$ receives an allocated quantity $y_{i}$ that is proportional to her order $x_{i}$, i.e.,

$$
y_{i}=\frac{x_{i}}{x_{i}+x_{-i}} \cdot K
$$

This is a simple rule commonly used in semiconductor manufacturing (Mallik and Harker, 2004), computing resources ( $\mathrm{Li}$ and $\mathrm{Li}, 2004$ ), and communication networks (Kelly, 1997). The retailers operate in independent markets, each with constant demand $D$. We assume that the demand is sufficiently large, i.e., $D>K / 2$; otherwise, each retailer can simply place an order to meet her entire demand and capacity allocation is no longer an issue.

Let the cost of each unit be $c$ and the market price be $p$. Then, given an allocated quantity $y_{i}$, the profit of retailer $i$ is $p \cdot \min \left\{D, y_{i}\right\}-c \cdot y_{i}$. The retailer earns the maximum possible profit of $(p-c) \cdot D$ when she secures an allocated quantity $y_{i}=D$. For convenience, we shall express profits relative to the above benchmark. Note that for every unit $y_{i}$ falls below demand $D$, retailer $i$ incurs a shortage cost of $s \equiv p-c$, and for every unit $y_{i}$ exceeds demand $D$, retailer $i$ incurs a wastage cost of $w \equiv c$. Therefore, the retailer's profit is $(p-c) \cdot D-w \cdot \max \left\{y_{i}-D, 0\right\}-s \cdot \max \left\{D-y_{i}, 0\right\}$. Too high an allocated quantity generates wastage costs while too low an allocated quantity leads to shortage costs.

With the above setting, we have a two-player game between the retailers. When retailer $i$ submits an order $x_{i}$ and her competitor submits an order $x_{-i}$, the payoff of retailer $i$ is given by

$$
\pi_{i}\left(x_{i}, x_{-i}\right)=(p-c) \cdot D-w \cdot \max \left\{\frac{x_{i} K}{x_{i}+x_{-i}}-D, 0\right\}-s \cdot \max \left\{D-\frac{x_{i} K}{x_{i}+x_{-i}}, 0\right\} .
$$

The above game does not have a finite pure-strategy Nash equilibrium because each player's best response is always to submit an order that is higher than her opponent's (see Cachon and Lariviere, 1999a). To circumvent this problem, we make a minor modification to our model. We assume that orders are constrained by an upper bound $U$, i.e., the retailers are not allowed to submit any order exceeding $U$. The assumption is reasonable in practice: when $U$ is large, orders above $U$ are not plausible. With this upper bound, it is easy to show that the unique Nash equilibrium in our capacity allocation game is $(U, U)$. In other words, both retailers submit the maximum allowed order of $U$ units and they both receive an equal allocated quantity of $K / 2$ units in equilibrium.

Interestingly, the Nash equilibrium prediction is invariant to model parameters. As long as they are in a capacity-constrained situation (i.e., $K<2 D$ ), in equilibrium, both retailers order $U$ units regardless of the shortage and wastage costs. Even if the upper bound $U$ is very large, both 
retailers always order exactly $U$ units - not one unit less. This is an extremely sharp prediction. In the next section, we shall proceed to conduct an experimental investigation of this prediction.

\section{Experiments and Results}

We adopt a between-subjects experimental design. There are two treatment conditions with different cost parameters as explained next. We use the following parameter values. The supplier's capacity is $K=90$ and each retailer faces demand $D=50$. (Note that the total demand is $2 D=100$ and exceeds capacity $K=90$.) The shortage cost is $s=5$. The above parameters remain unchanged across the two cost conditions. However, the wastage cost is chosen as $w=2$ in one condition and $w=20$ in another. We refer to the former as the low-cost condition and the latter as the high-cost condition. The order quantities of both retailers are restricted to be integers between 0 and $U$, where $U=100$. During the experiment, subjects earned points as their payoffs. The payoff (in points) for a subject whose allocated quantity is $y$ is: $100-s \cdot \max \{D-y, 0\}-w \cdot \max \{y-D, 0\}$. In our experiment, we shifted the payoff function by a constant so that the maximum possible profit for all treatment conditions is always 100 points.

We conducted one experimental session for each of the two treatments. In each session, there were 30 subjects, all of whom are undergraduates at a major university in China. Each session consisted of 30 decision rounds. In each round, every subject is randomly matched with another subject and the pair plays the capacity allocation game, so each subject played the game 30 times in total. Subjects are matched with every other subject at most twice, and they never know the identity of their opponents. At the beginning of a session, the administrator explained to the subjects the experimental instructions, as shown in the Appendix A. Then, subjects were assigned a set of exercises to ensure their understanding of the task. Each subject was required to obtain a perfect score on the exercise before starting the decision rounds. Each session lasted for about 60 minutes. When a session ended, the total points earned by each subject over all rounds were summed and converted to cash payments. The average earning of each subject is approximately 50 RMB, which is equivalent to US\$15 after adjusting by purchasing power parity.

We present some summary statistics of our experimental results. Figure 1 shows the distribution of subjects' orders as well as the Nash equilibrium prediction, for both high-cost (left panel) and low-cost (right panel) sessions. There is a clear discrepancy between our data and the predictions by the Nash equilibrium that both retailers will order 100 units for both cost conditions. In the high-cost session, there are only 122 observations (13.56\% of a total of 900 observations) that coincide with the Nash equilibrium prediction; for the remaining $86.44 \%$ of our data, subjects' 
behavior goes against standard game-theoretic reasoning. Similarly, in the low-cost session, only 723 out of 900 observations (80.33\%) agree with the Nash equilibrium prediction. Although this is higher than the corresponding figure of $13.56 \%$ in the high-cost session, our results suggest that the predictive power of Nash equilibrium remains inadequate. There remains a significant proportion (about 20\%) of the data that is inconsistent with the Nash equilibrium prediction.
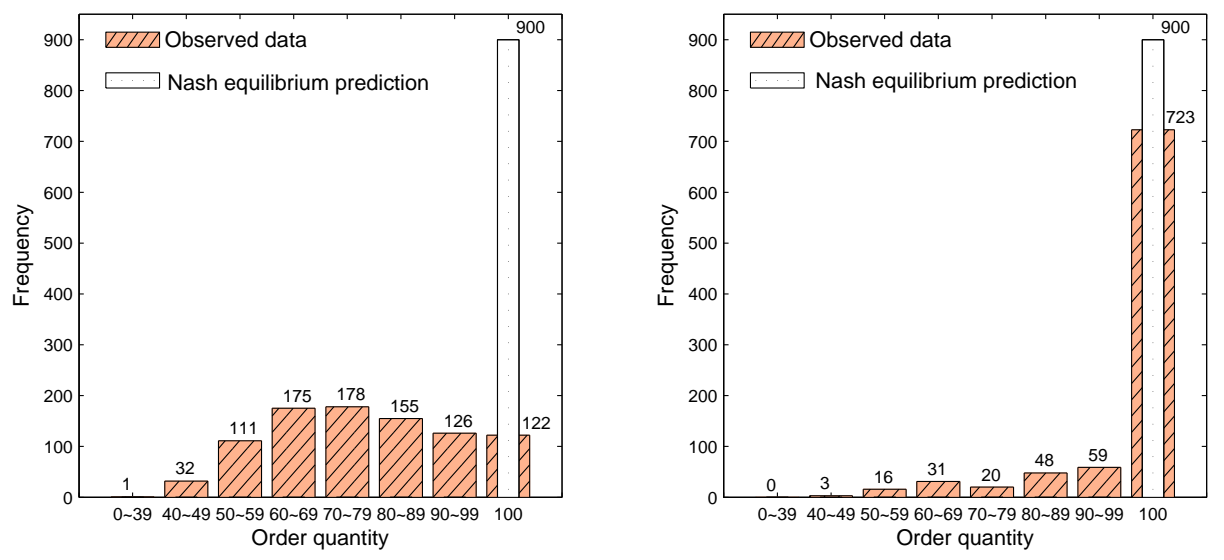

Figure 1: Distribution of orders in the high-cost condition (left) and low-cost condition (right), compared to the Nash equilibrium prediction

Next, our data show systematic differences between the low-cost and high-cost conditions. For example, the average order is 75.8 in the high-cost condition but it increases to 95.8 in the low-cost condition. Furthermore, as many as $86.89 \%$ of the orders exceed 90 in the low-cost condition but there is only $27.56 \%$ in the high-cost condition. Similarly, on the other extreme, only $2.11 \%$ of the orders are less than 60 in the low-cost condition but the corresponding figure of $16.00 \%$ is much higher in the high-cost condition. These observations suggest that subjects tend to order more in the low-cost condition. However, the Nash equilibrium predicts that subjects always order 100 units regardless of model parameters.

Finally, our data also exhibit systematic time trends. In our experiment, each subject has the chance to play the game 30 times. The average orders chosen by subjects in each round are shown in Figure 2, together with the 5-th and 95-th percentiles of the orders in each round. From the figures, we see a clear upward trend in orders over time. For example, in the high-cost condition (left panel), the average order is 53.5 in Round 1 but increases to 85.6 by Round 30; similarly, in the low-cost condition (right panel), the average order is 75.2 in Round 1 but increases to 98.3 by Round 30. As retailers increase their order quantities, their choices move toward the Nash 
equilibrium prediction of $U=100$. Our results suggest that subjects are learning as they play the game repeatedly.
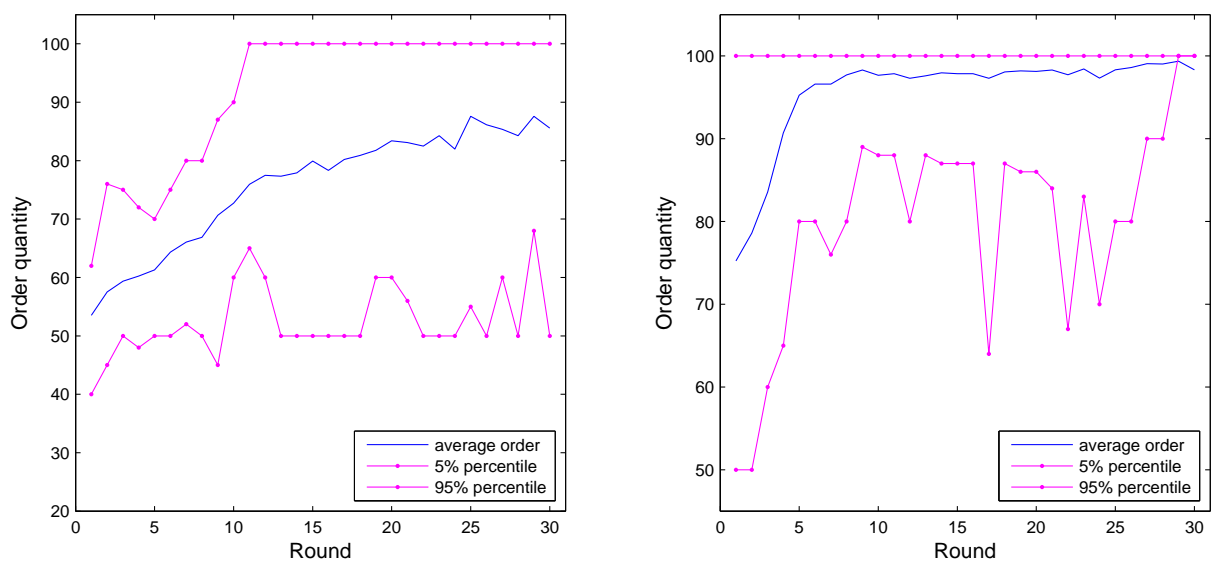

Figure 2: Time trends in the data for the high-cost condition(left) and low-cost condition(right)

In summary, our experimental results highlight three main empirical regularities that the Nash equilibrium prediction fails to account for. First, subjects frequently submit orders that are smaller than the Nash equilibrium prediction. Second, the deviation described above appears to be more significant in the high-cost condition compared to the low-cost condition. Third, there are significant time trends: the fraction of observations that coincide with the Nash equilibrium increases over time.

In the next section, we shall explain these observations using a behavioral decision model.

\section{A Model of Bounded Rationality}

In standard game-theoretic reasoning, there is common knowledge of perfect rationality, which entails two assumptions. First, every player is a perfect optimizer and is able to choose the best response to her opponents' actions. Second, every player can perfectly predict her opponents' choices because her opponents are also perfectly rational and thus they choose their best responses accordingly. These assumptions give rise to the Nash equilibrium concept, which often yields sharp theoretical predictions. However, in practice, common knowledge of perfect rationality seldom holds.

McKelvey and Palfrey (1995) proposed the idea of a Quantal Response Equilibrium (QRE), which provides a way to incorporate bounded rationality into game-theoretic reasoning. Formally, a QRE with two players can be defined as follows. Let $\pi_{i}\left(a_{i}, a_{-i}\right)$ denote player $i$ 's payoff from 
choosing action $a_{i}$ when her opponent chooses $a_{-i}$. Then, the QRE is a strategy profile in which player $i$ 's action $A_{i}$ is a random variable defined over action set $S_{i}$ and her opponent's action $A_{-i}$ is also a random variable defined over action set $S_{-i}$. For player $i$, each admissible action $a_{i} \in S_{i}$ is chosen with probability

$$
P_{i}\left(a_{i}\right)=\frac{\exp \left\{E \pi_{i}\left(a_{i}, A_{-i}\right) / \beta\right\}}{\sum_{a_{i}^{\prime} \in S_{i}} \exp \left\{E \pi_{i}\left(a_{i}^{\prime}, A_{-i}\right) / \beta\right\}} .
$$

These choice probabilities are obtained by assuming that each player chooses a "noisy" best response by maximizing $E \pi_{i}\left(a_{i}, A_{-i}\right)+\varepsilon_{i}$ instead of maximizing $E \pi_{i}\left(a_{i}, A_{-i}\right)$. We obtain the logit specification above by assuming that the noise terms $\varepsilon_{i}$ are i.i.d. with an extreme-value distribution (McFadden, 1981). ${ }^{1}$ For the opponent, each admissible action $a_{-i}$ is also chosen with probability given by a similar expression as (3).

Note that the QRE embeds a quantal choice model into each individual player's decision framework. In this way, the QRE captures bounded rationality in every player's behavior. It satisfies two important properties. First, rather than perfectly optimizing payoffs, players choose stochastic best responses. They are noisy optimizers because all feasible actions are chosen with strictly positive probability, although actions with higher expected payoffs are chosen more often. Second, players face uncertainty over their opponents' choices because they recognize that their opponents are also playing stochastic best responses. Similar to the Nash equilibrium, the QRE is internally consistent in the sense that players have rational expectations and possess correct beliefs of the probability distributions over opponents' actions.

The QRE model has a single parameter $\beta$. We call this the bounded rationality parameter. For sake of parsimony, we use a common parameter $\beta$ for every player; Rogers et al. (2009) incorporate heterogeneity in players' bounded rationality but we leave this for future research. We interpret the magnitude of the parameter $\beta$ as the extent of bounded rationality in each player's behavior. Specifically, it reflects the extent of cognitive and computational limitation of the player. When $\beta \rightarrow \infty$, the player lacks the ability to make any rational judgement and thus randomizes over all alternatives with equal probabilities. On the other hand, when $\beta \rightarrow 0$, the player chooses the payoff-maximizing alternative with certainty, i.e., the QRE in this limiting case is consistent with the Nash equilibrium. In this case, if there is more than one optimal choice, the player uniformly randomizes among them.

\footnotetext{
${ }^{1}$ Haile et al. (2008) caution that if the error terms $\varepsilon_{i}$ are allowed to be correlated or have different marginal distributions, the QRE imposes no falsifiable restrictions on data. But, they note that "the standard logit specification imposes such restrictions and can starkly limit the set of possible QRE outcomes in many games (p. 196)."
} 
We now apply the QRE framework to analyze the capacity allocation game. Under the proportional allocation rule, retailer $i$ receives allocated quantity $y_{i}=\frac{x_{i}}{x_{i}+x_{-i}} \cdot K$ and the resulting payoff function $\pi_{i}\left(y_{i}\right)$ is

$$
\pi_{i}\left(y_{i}\right)=M-s \cdot \max \left\{D-y_{i}, 0\right\}-w \cdot \max \left\{y_{i}-D, 0\right\}
$$

where $M$ is the maximum possible profit of each player. Note that the QRE does not change as $M$ varies. This is because the expected profit from each possible choice is shifted by the same amount that does not change the relative payoffs and thus does not change the choice probabilities in equation (3). Thus, in our experiment, we can exogenously fix $M$ to be any arbitrary constant, while $K, D, s, w$ are set as parameters that can be manipulated.

We provide some numerical examples with parameters $K=90, D=50, s=5$, and $w=20$, as in the high-cost condition in our experiment. Figure 3 presents the QRE distributions with different values of $\beta$. These choice distributions suggest that the QRE model can explain our data better since it allows for choices below the Nash equilibrium prediction of 100 . As $\beta$ increases, the distribution shifts towards the left and eventually converges to the uniform distribution. As $\beta$ decreases, the QRE distribution becomes more concentrated around 100 and converges toward a point mass at the Nash equilibrium prediction of 100. In this way, the bounded rationality parameter $\beta$ provides flexibility in model fitting.

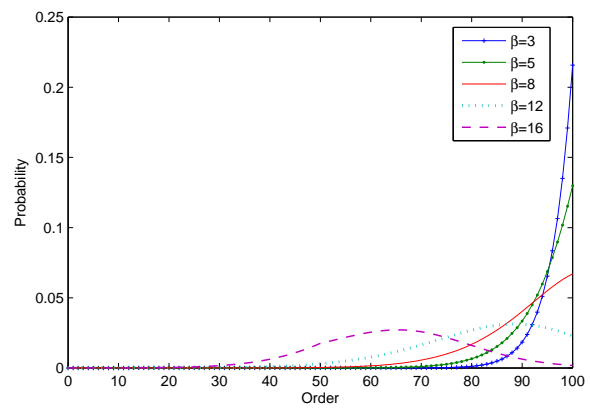

Figure 3: QRE distributions for the high-cost condition with different values of $\beta$

Similarly, we numerically compute the QRE for the low-cost condition in our experiment, with parameters $K=90, D=50, s=5$, and $w=2$. Note that the high-cost condition and the low-cost condition differ only in $w$. For the same $\beta$, it is interesting to compare the QRE distributions under the two different cost conditions. For example, Figure 4 presents the two QRE distributions for the case where $\beta=12$. The figure shows that even with the same value of $\beta$, the QRE distributions may change with the cost parameter $w$. Particularly, for the low-cost condition, choices tend to be 
more clustered around 100. This is one of the empirical regularities observed in our experiment. On the other hand, recall that the Nash equilibrium yields the same prediction for both cost conditions.

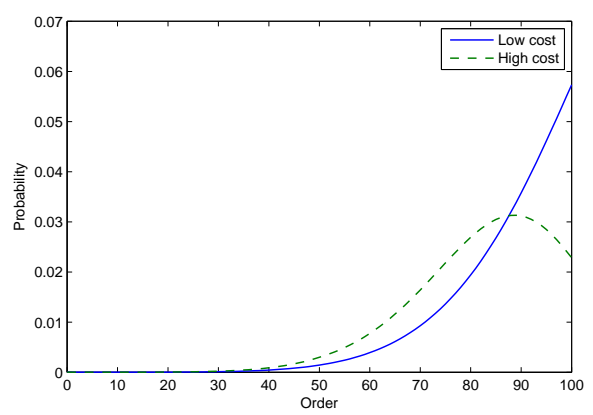

Figure 4: QRE distributions for the high-cost and low-cost conditions with $\beta=12$

The numerical examples above show that the QRE model may be a good candidate to help explain our experimental observations. However, the model has a shortcoming. All the examples in Figures 3 and 4 yield unimodal QRE distributions, either with a bell-shape or with a peak at 100. In contrast, the data (see Figure 1) may exhibit two peaks, one at 100 and another around the 60-69 range. Thus, the restriction to unimodal distributions may compromise the performance of the QRE model and it is always in theory possible to find another model that fits the data better. Therefore, our primary goal is not to find the best-fitting model, but simply to understand how much better the QRE performs compared to the Nash equilibrium benchmark.

In addition, we enrich the standard QRE model by allowing the bounded rationality parameter $\beta$ to change over time. In our experiment, the data suggest that players become "more rational" through repeated game play because their orders increase and converge toward the Nash equilibrium prediction. This phenomenon indicates that the bounded rationality parameter $\beta$ may in fact decrease over time due to learning. To incorporate such an effect, we allow the bounded rationality parameter in round $t$, denoted by $\beta(t)$, to decay exponentially over time:

$$
\beta(t)=\beta+(\alpha-\beta) e^{-\delta(t-1)}
$$

Similar approaches have been adopted in the literature (e.g., McKelvey and Palfrey, 1992). In particular, we have $\beta(1)=\alpha$ and $\beta(\infty)=\beta$, so we may interpret $\alpha$ and $\beta$ respectively as the initial and eventual bounded rationality parameters, and $\delta$ as the rate of learning. To reflect the learning patterns observed in the data, we expect the value of $\alpha$ to be larger than the value of $\beta$, so $\beta(t)$ would decrease from $\alpha$ towards $\beta$ as the round $t$ goes from 0 to $\infty$. 


\section{Maximum Likelihood Estimation}

In this section, we fit the model to our data from the experiment. We use the principle of maximum likelihood to obtain parameter estimates and interpret subjects' behavior in the experiment.

We specify the likelihood function and describe how it depends on our model parameters. In the estimation, we fit up to four parameters: the initial bounded rationality $\alpha$, the eventual bounded rationality $\beta$, the learning rate $\delta$, and a noise term $\epsilon$ (explained below). Given parameters $\alpha, \beta$ and $\delta$, let $f_{t}(x)$ be the probability of choosing an order quantity $x$ in round $t$. According to the QRE model described by equation (3), it follows that

$$
f_{t}(x)=\frac{\exp \left\{E \pi\left(x, R_{t}\right) / \beta(t)\right\}}{\sum_{x^{\prime} \in S} \exp \left\{E \pi\left(x^{\prime}, R_{t}\right) / \beta(t)\right\}} .
$$

Here, $\beta(t)$ is the bounded rationality parameter in round $t$ as given by equation (5), and $R_{t}$ is a random variable with distribution $\left\{f_{t}(\cdot)\right\}$ over the choice domain $S=\{0,1, \ldots, 100\}$. Since the capacity allocation game is symmetric and we use the same bounded rationality parameter for both retailers, the same QRE distribution will apply to both of them. Let $X=\left\{x_{i t} \mid i=1,2, \ldots I ; t=\right.$ $1,2, \ldots, T\}$ be the order quantities in the experimental data, where $x_{i t}$ represents the order chosen by subject $i$ in round $t, I$ is the total number of subjects, and $T$ is the total number of decisions rounds in a session. Then, we have the following likelihood function:

$$
L(\alpha, \beta, \delta, \epsilon \mid X)=\prod_{i=1}^{I} \prod_{t=1}^{T}\left\{(1-\epsilon) \cdot f_{t}\left(x_{i t}\right)+\epsilon \cdot \frac{1}{|S|}\right\},
$$

that is, with probability $(1-\epsilon)$, the player chooses an order according to the QRE distribution $\left\{f_{t}(\cdot)\right\}$, and with probability $\epsilon$, the player uniformly chooses an order over the choice domain $S=\{0,1, \ldots, 100\}$ so that the conditional probability of observing $x_{i t}$ is $1 /|S|$ with $|S|=101$. The adoption of a uniform error term $\epsilon$ is common in experimental economics (see, e.g., Harless and Camerer, 1994). If the specified model fits the data perfectly, we would have $\epsilon=0$. In practice, we expect $\epsilon$ to be positive but small. To fit the model, we maximize the likelihood function $L(\cdot)$ over the four parameters $\alpha, \beta, \delta, \epsilon$.

There are several special cases of our model that deserve special attention. First, when we restrict $\alpha=\beta=\delta=0$ (hence $\beta(t) \equiv 0$ over all $t$ ), the QRE reduces to the Nash equilibrium. In this case, there is only a single free parameter $\epsilon$. Using the generalized likelihood principle, we may check whether the above three parameters $(\alpha, \beta$ and $\delta)$ are indeed zero; if not, the data would provide evidence in favor of bounded rationality (in the form of QRE). Second, when we restrict $\alpha=\beta$ and $\delta=0$ (hence $\beta(t) \equiv \beta$ over all $t$ ), there is no learning because the bounded rationality 
parameter would remain constant over all rounds. We call the second case the static model, which has two parameters $\beta$ and $\epsilon$. This two-parameter special case can be used to detect learning effects. Third, we may also restrict $\beta=0$ (hence $\beta(t)=\alpha e^{-\delta(t-1)}$ ). This special case allows for bounded rationality to be present initially (through the parameter $\alpha$ ) but assumes that choice behavior would eventually approach perfect rationality and match the Nash equilibrium prediction. Thus, we term this special case the transient model, which has three parameters $\alpha, \delta$ and $\epsilon$. We use this special case to check whether boundedly rational behavior is persistent over time.

Table 1: Estimation result of high-cost condition

\begin{tabular}{|c|c|c|c|c|}
\hline Theoretical model & Nash & Static & Transient & Full Model \\
\hline$\alpha$ & - & - & 18.745 & 20.912 \\
\hline$\beta$ & - & 13.410 & - & 8.260 \\
\hline$\delta$ & - & - & 0.027 & 0.079 \\
\hline$\epsilon$ & 0.873 & 0.004 & 0.004 & 0.004 \\
\hline Number of parameters & 1 & 2 & 3 & 4 \\
\hline Log-likelihood & -3940.0 & -3720.1 & -3465.3 & -3460.4 \\
\hline Likelihood ratio test against Full model & $\begin{array}{c}\chi^{2}=959.2 \\
(p=0.000)\end{array}$ & $\begin{array}{c}\chi^{2}=519.4 \\
(p=0.000)\end{array}$ & $\begin{array}{c}\chi^{2}=9.8 \\
(p=0.002)\end{array}$ & - \\
\hline
\end{tabular}

We first fit the models using data from the high-cost session. All numerical computations are performed using Matlab. Table 1 reports the parameter estimates for our full QRE model as well as the special cases described above. We emphasize three observations from Table 1.

1. The log-likelihood score of the Nash equilibrium model is significantly smaller than those of the other three models. The likelihood ratio test between the Nash model and any of other three models yields $\chi^{2}$-statistics in excess of 100 ( $p$-value $\left.<0.0001\right)$, suggesting that all model parameters $\alpha, \beta$, and $\delta$ are significantly positive. This result indicates that the bounded rationality models (based on QRE) fit our experimental data better than the model of perfect rationality (based on Nash equilibrium). In fact, for the Nash equilibrium model, the maximum likelihood estimate of the noise parameter is $\epsilon=0.873$, which is much larger than the corresponding estimates of other models. This suggests that the Nash equilibrium model fits the data poorly and thus gives rise to a large noise parameter.

2. The static model, which assumes no learning, yields a log-likelihood score of -3720.1 , while the full model yields a log-likelihood score of -3460.4 . The likelihood ratio test statistic is 
$\chi^{2}=519.4(p$-value $<0.0001)$, so the estimates of $\alpha$ and $\delta$ are statistically significant. This result provides evidence of learning in the data. ${ }^{2}$

3. Comparing the last two columns of Table 1, we see that the log-likelihood scores of the full model and the transient model are -3460.4 and -3465.3 respectively. The likelihood ratio test yields $\chi^{2}=9.8(p$-value $=0.0017)$, which suggests that $\beta$ (interpreted as a measure of bounded rationality that persists after repeated learning) is significantly positive. This implies that the bounded rationality parameter will eventually converge to a positive value of 8.260 , though convergence is not necessarily guaranteed after the 30 rounds of game play in the data. Therefore, our result shows that although subjects are learning through repeated game play, bounded rationality remains a persistent phenomenon.

Next, Table 2 presents the analogous results for the low-cost session. We make the following three observations.

1. Similar to our observation from the high-cost condition, the Nash equilibrium model performs the worst. In particular, compared to the full model (with log-likelihood of -1249.2), the Nash equilibrium model (with log-likelihood of -1261.3$)$ is strongly rejected $\left(\chi^{2}=24.2, p\right.$-value $<0.0001)$. This suggests that the bounded rationality is again significant in the low-cost condition. However, the evidence appears weaker since the test-statistics are now smaller, though still highly significant.

2. Similar to before, we again find some evidence of learning, although somewhat weaker. For example, comparing the middle two columns, we see that the two-parameter static model without learning is rejected in favor of the three-parameter transient model with learning $\left(\chi^{2}=5.2, p\right.$-value $\left.=0.0225\right)$.

3. We find no evidence for the persistence of bounded rationality in the low-cost condition. In the full model, maximum likelihood estimation yields $\beta=0$, i.e., bounded rationality eventually vanishes. This result is different from the high-cost condition.

\footnotetext{
${ }^{2}$ To check the robustness of our result, we consider an alternative learning curve of the form $\beta(t)=\beta+\frac{\alpha}{1+\delta(t-1)}$; see, e.g., De Bruyn and Bolton (2008). Compared to our learning equation in (5), which assumes exponential discounting of the bounded rationality parameter over time, their specification assumes hyperbolic discounting. With the alternative learning curve, the static no-learning model is again rejected in favor of the full model $\left(\chi^{2}=520.0\right.$, $p$-value $<0.0001)$.
} 
Table 2: Estimation result of low-cost condition

\begin{tabular}{|c|c|c|c|c|}
\hline Theoretical model & Nash & Static & Transient & Full Model \\
\hline$\alpha$ & - & - & 0.250 & 0.250 \\
\hline$\beta$ & - & 0.161 & - & 0.000 \\
\hline$\delta$ & - & - & 0.029 & 0.029 \\
\hline$\epsilon$ & 0.199 & 0.189 & 0.187 & 0.187 \\
\hline Number of parameters & 1 & 2 & 3 & 4 \\
\hline Log likelihood & -1261.3 & -1251.8 & -1249.2 & -1249.2 \\
\hline Likelihood ratio test against Transient model & $\begin{array}{c}\chi^{2}=24.2 \\
(p=0.000)\end{array}$ & $\begin{array}{c}\chi^{2}=5.2 \\
(p=0.023)\end{array}$ & - & - \\
\hline
\end{tabular}

Our analysis thus far is encouraging. The structural estimation results suggest that a model of bounded rationality in the form of QRE can provide a good fit of the data. ${ }^{3}$ Although our experimental investigation yields several anomalies that cannot be explained by the standard Nash equilibrium prediction, we have shown that the QRE provides an appealing alternative.

In Figure 5, we compare the predictions of our full model with the data; we present plots for Rounds 1-10 (top), Rounds 11-20 (middle) and Rounds 21-30 (bottom), in the high-cost (left) and low-cost (right) sessions. Dividing the data and predictions this way, we see that the QRE model appears to fit our data reasonably well, both over time and over the two cost conditions.

To conclude this section, we raise an open question. What determines the level of bounded rationality and how does it vary across games? In our analysis above, we have seen how estimates of the bounded rationality parameter $\beta$ can vary substantially between the high cost and low cost conditions. Why is this so? In general, some games may be more complex than others. When playing "simpler" games, people may be able to behave "more rationally" and thus the bounded rationality parameter may be smaller. A comprehensive theory that systematically identifies how the level of bounded rationality $\beta$ depends on the specific problem context (e.g., rules of the game) will be extremely useful because it will allow us to generate precise predictions on outcomes without first having to estimate $\beta$. We believe this is an important issue for future research. The capacity allocation setting in this paper may provide a useful starting point: setting different allocation rules

\footnotetext{
${ }^{3}$ To guard against data overfitting, we repeat our analysis using three-fold cross validation. In the high-cost condition, we obtain log-likelihood scores for our 4 models of $-3953.3,-3739.4,-3496.4,-3474.5$, which provide even stronger support for the full model $\left(\chi^{2}=43.8, p\right.$-value $\left.<0.0001\right)$. However, in the low-cost condition, we obtain the log-likelihood scores of $-1283.2,-1277.4,-1277.6,-1278.3$, suggesting that learning is not significant but the QRE model is still preferred over Nash $\left(\chi^{2}=11.6, p\right.$-value $\left.<0.001\right)$.
} 

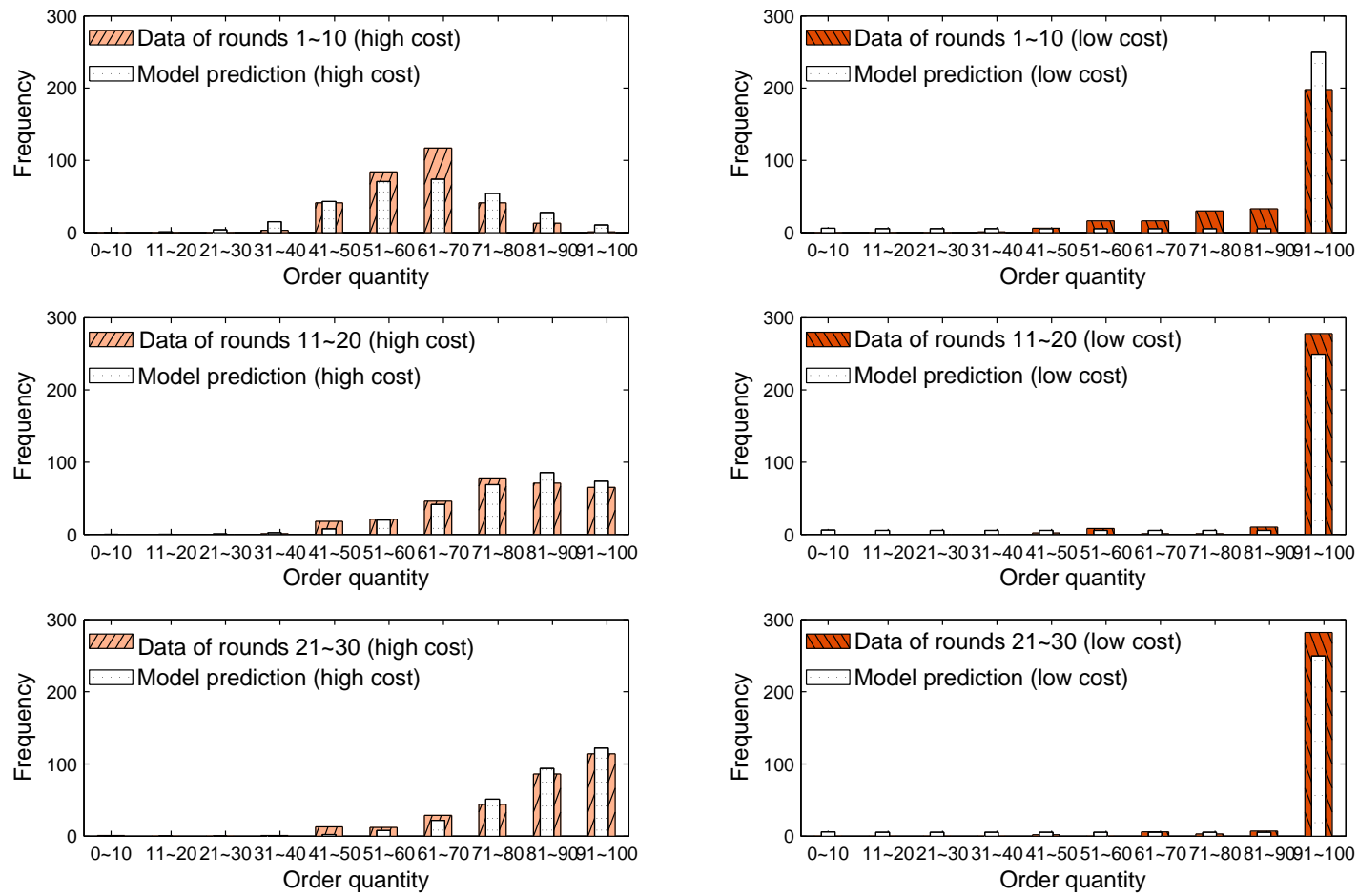

Figure 5: Comparison between the experimental data and the full model predictions in the highcost condition (left) and low-cost condition (right), for Rounds 1-10 (top), Rounds 11-20 (middle) and Rounds 21-30 (bottom)

allow us to study different games while remaining within a common operational setting. Doing so isolates the contextual effects without potentially introducing other confounding factors.

\section{Alternative Explanations}

We have seen that the QRE model gives more accurate and nuanced predictions of our capacity allocation games compared to Nash equilibrium. However, there could be alternative explanations of the data. In this section, we consider several alternatives and compare them to the QRE. Our results suggest that it is not easy to find a simple and compelling explanation of the data, and the QRE model appears to be an attractive candidate.

\subsection{Modified utility functions}

One of the most direct way of perturbing a model to generate different predictions is to modify the underlying utility functions. In the capacity allocation games, our experimental data show that 
subjects order substantially smaller quantities than the Nash equilibrium when players maximize expected payoffs. If players instead seek to maximize some other utility function, would they choose to order smaller quantities in equilibrium? For example, players may be loss-averse and hence may shy away from placing large orders that may generate negative payoffs.

To test this conjecture, we consider several alternative utility functions. Specifically, we consider players who may be averse to (i) losses, (ii) leftover inventory, (iii) shortages, and (iv) inventory errors. We model the above using a piecewise linear utility term, similar to Schweitzer and Cachon (2000) and Ho, Lim, and Cui (2010). We find that under these reference-dependent utility functions, the Nash equilibrium $x^{*}=100$ remains unchanged. Details are in Appendix B.

The above results suggest that our capacity allocation game is a useful vehicle for identifying bounded rationality behavior. Since the Nash equilibrium is extremely robust to alternative utility specifications, the deviations that we observe require another explanation, such as the QRE.

\subsection{Incomplete information}

Another plausible explanation for why subjects do not play the Nash equilibrium is because they do not trust their opponents to do so. In our capacity allocation game, the Nash equilibrium $x^{*}=100$ is the best response to itself. However, if the opponent chooses another quantity (e.g., 50 due to demand being 50), then ordering 100 may in fact be a very bad thing to do and thus the player should place a smaller order instead. Such reasoning may justify the under-ordering observed in our data.

To formalize this intuition, we extend our model to incorporate private information over player types. There are two types of players: strategic and non-strategic. With probability $p$, the player is perfectly rational, and with probability $1-p$, the player always orders exactly equal to demand (i.e., 50); we feel that truth-telling is a natural type of non-strategic behavior in capacity allocation games. With this setup, we have a game of incomplete information.

In the Nash equilibrium, non-strategic players always order 50 (by assumption) while strategic players may order either 100 or 62 . Specifically, when $p$ is sufficiently large, strategic players order 100 (i.e., the best response to strategic players) and when $p$ is sufficiently small, strategic players order 62 (i.e., the best response to non-strategic choice of 50 units). Detailed calculations are shown in Appendix B. Although this model admits choices below 100, it does not accommodate choices between 62 and 100, which account for more than $60 \%$ of our data in the high-cost session. ${ }^{4}$

\footnotetext{
${ }^{4}$ In theory, it is always possible to "fill in the gaps" by introducing additional player types and specifying their preferences so that their equilibrium orders fall between 62 and 100. However, doing so increases model complexity because we would have to specify both the probability and preferences of each type.
} 
Therefore, we conclude that incomplete information does not provide a satisfactory explanation of the data.

\subsection{Iterative thinking}

Finally, we consider another possible explanation of why subjects do not play the Nash equilibrium, based on models of iterative thinking. In these models, players repeatedly perform best response calculations; these calculations may converge to the Nash equilibrium, but if players terminate this process prematurely they may make non-Nash choices. This type of player behavior forms the basis of level- $k$ models (Stahl and Wilson, 1995) and cognitive hierarchy models (Camerer et al., 2004). We check whether limited iterative thinking can provide an alternative explanation for our data.

In capacity allocation games, one form of non-strategic behavior could be ordering the true demand (i.e., choosing $x=50$ ). Following the terminology of Stahl and Wilson (1995), we call such players level-0 players. Applying level- $k$ models, we assume that level- $k$ players best-respond to level- $(k-1)$ players, so players of sufficiently high levels play the Nash equilibrium. In our capacity allocation games, letting $L_{k}$ denote the choices of level- $k$ players, it is easy to see that $L_{0}=50$, $L_{1}=62, L_{2}=77, L_{3}=96$, and $L_{n}=100$ when $n \geq 4 .^{5}$ Then, choosing a level- $k$ model to explain our experimental data comes down to finding a distribution of player types. Specifically, let $p_{k}$ denote the probability of level- $k$ players in the population. Then, the level- $k$ model predicts that with probability $p_{k}$, the quantity $L_{k}$ will be chosen.

It is clear that limited iterative thinking models can accommodate choices below the Nash equilibrium of 100. However, only orders within a relatively small discrete set of choices, e.g., $\{50,62,77,96,100\}$, are admissible, and all other choices in between can only be explained using a noise term $\epsilon$. In contrast, the QRE model allows for all choices below 100. This comparison suggests that the QRE provides a simple but compelling way to explain the observed data. ${ }^{6}$

\section{Conclusion}

We conduct laboratory experiments of capacity allocation games and identify several key empirical regularities in the data. Specifically, subjects tend to order much less than the Nash equilibrium

\footnotetext{
${ }^{5}$ Alternatively, level- $k$ players can best-respond to all lower types (see Camerer et al., 2004). In our capacity allocation game, this implies that the choices $L_{k}$ increase in $k$ more slowly but may converge to $L_{\infty}<100$.

${ }^{6}$ We fit the level- $k$ model to the data using maximum likelihood as outlined in Section 5 . Despite having a larger number of parameters (since we allow for a general distribution of thinking levels), the level- $k$ model yields log-likelihood scores of -3894.9 and -1258.1 for the high-cost and low-cost data. In other words, the level- $k$ model performs worse than our static model, with corresponding likelihood scores of -3720.1 and -1251.8 .
} 
prediction, although they do learn to adjust their orders upward over time, and the magnitude of disparities relative to the Nash equilibrium prediction appears to be more substantial in the highcost condition. Our goal is to develop a descriptive model that explains subjects' ordering behavior.

We use the QRE framework to fit our experimental data, which is based on the premise that players stochastically "better-respond" (rather than "best-respond") to their decision environments. They do not always choose the optimal alternative, but more attractive alternatives are chosen more frequently. In a game-theoretic setting, when all players stochastically respond to one another's actions, we have a QRE. The QRE integrates two important strategic considerations - inability to perfectly optimize one's own actions and lack of absolute certainty over opponents' actions into a unified framework involving decision errors and noise. We find that the QRE framework is versatile. When appropriately calibrated, it fits our data reasonably well. When we test several nested special cases (including the Nash equilibrium), we find evidence for suboptimal decisionmaking, learning through repeated game play, as well as persistence of bounded rationality over time. Finally, we ask the question: could there be alternative behavioral models that explain our data equally well, if not better? To this end, we consider several alternative behavioral decision models that incorporate modified utility functions, incomplete information, and limited iterative thinking. Our results indicate that the QRE model provides a simple but compelling explanation of the behavior observed in the data.

We conclude this paper with two thoughts. First, we feel that in operations models, gametheoretic analysis may sometimes yield implausible predictions. For example, in queueing admission games (e.g., Naor, 1969), equilibrium behavior has a threshold structure in the sense that customers always join or always balk depending on whether queue length is above or below a threshold. Similarly, a more recent example in the context of strategic customer behavior in buy-or-wait games (see Su and Zhang, 2008) suggests that customers either all buy or all wait, depending on model parameters. This type of bang-bang behavior seems too extreme to be true empirically. In such situations, we feel that the QRE can be a natural alternative platform for rigorous analysis. In some cases, we speculate that the QRE may even generate fresh insights. Second, although we have in this paper focused on the capacity allocation game, we remind readers that the QRE framework is simple yet general and can be readily applied to other operations contexts such as those mentioned above. In some sense, as long as the modeling strategy involves choices and payoff functions, we can always infuse the model with decision noise to study the impact of bounded rationality in the form of QRE. 


\section{Acknowledgements:}

This research is supported by NSF of China under Grants 71031005 and 70871066.

\section{References}

[1] Anderson, S. P., J. K. Goeree, C. A. Holt. 1998. Rent seeking with bounded rationality: An analysis of the all-pay auction. Journal of Political Economy 106(4) 828-853.

[2] Anderson, S. P., J. K. Goeree, C. A. Holt. 2001. Minimum-effort coordination games: Stochastic potential and logit equilibrium. Games and Economic Behavior 34(2) 177-199.

[3] Anderson, S. P., J. K. Goeree, C. A. Holt. 2002. The logit equilibrium: A perspective on intuitive behavioral anomalies. Southern Economic Journal 69(1) 21-47.

[4] Cachon, G., M. Lariviere. 1999a. An equilibrium analysis of linear, proportional and uniform allocation of scarce capacity. IIE Transactions 31(9) 835-849.

[5] Cachon, G., M. Lariviere. 1999b. Capacity choice and allocation: Strategic behavior and supply chain performance. Management Science 45(8) 1091-1108.

[6] Cachon, G., M. Lariviere. 1999c. Capacity allocation using past sales: When to turn-and-earn. Management Science 45(5) 685-703.

[7] Camerer, C.F., T.-H. Ho, J.-K. Chong. 2004. A cognitive hierarchy model of games. Quarterly Journal of Economics. 119(3) 861-898

[8] Capra, C. M., J. K. Goeree, R. Gomez, C. A. Holt. 1999. Anomalous behavior in a traveler's dilemma? American Economic Review 89(3) 678-690.

[9] Chen, H.-C., J.W. Friedman, J.-F. Thisse. 1997. Boundedly rational Nash equilibrium: A probabilistic choice approach. Games and Economic Behavior 18(1) 32-54.

[10] De Bruyn, A., G.E. Bolton. 2008. Estimating the influence of fairness on bargaining behavior. Management Science 54(10) 1774-1791.

[11] Dewan, S., H, Mendelson. 1990. User delay costs and internal pricing for a service facility. Management Science 36(12) 1502-1517.

[12] Fey, M., R. D. McKelvey, T. R. Palfrey. 1996. An experimental study of constant-sum centipede games. International Journal of Game Theory 25(3) 269-287. 
[13] Goeree, J. K., C. A. Holt. 2000. Asymmetric inequality aversion and noisy behavior in alternating-offer bargaining games. European Economic Review 44(4-6) 1079-1089.

[14] Goeree, J. K., C. A. Holt. 2001. Ten little treasures of game theory and ten intuitive contradictions. American Economic Review 91(5) 1402-1422.

[15] Goeree, J. K., C. A. Holt, T. R. Palfrey. 2002. Quantal response equilibrium and overbidding in private-value auctions. Journal of Economic Theory 104(1) 247-272.

[16] Haile, P. A., A. Hortacsu, G. Kosenok. 2008. On the empirical content of quantal response equilibrium. American Economic Review 98(1) 180-200.

[17] Harless, D. W., C. F. Camerer. 1994. The predictive utility of generalized expected utility theories. Econometrica 62(6) 1251-1289.

[18] Ho, T.-H., J.J. Zhang. 2008. Designing price contracts for boundedly rational customers: Does the framing of the fixed fee matter? Management Science 54(4) 686-700.

[19] Ho, T.-H., N. Lim, T. H. Cui. 2010. Reference dependence in multilocation newsvendor models: A structural analysis. Management Science 56(11) 1891-1910.

[20] Kelly, F. 1997. Charging and rate control for elastic traffic. European Transactions on Telecommunications. 8(1) 33-37.

[21] Lee, H. L., V. Padmanabhan, S. Whang. 1997. Information distortion in a supply chain: the bullwhip effect. Management Science 43(4) 546-558.

[22] Li, C., L. Li. 2004. Competitive proportional resource allocation policy for computational grid. Furture Gerneration Computer System 20 1041-1054.

[23] Lim, N., T.-H. Ho. 2007. Designing price contracts for boundedly rational customers: Does the number of blocks matter? Marketing Science 26(3) 312-326.

[24] Luce, R. D. 1959. Individual choice behavior: A theoretical analysis. Wiley, New York.

[25] Mallik, S., P. T. Harker. 2004. Coordinating supply chains with competition: Capacity allocation in semiconductor manufacturing. Eur. J. Oper. Res. 159(2) 330-347.

[26] McFadden, D. 1981. Econometric models of probabilistic choice. C. F. Manski and D. McFadden, eds. Structural Analysis of Discrete Data with Econometric Applications. MIT Press, Cambridge, MA, 198-272. 
[27] McKelvey, R. D., T. R. Palfrey. 1992. An experimental study of the centipede game. Econometrica 60(4) 803-836.

[28] McKelvey, R. D., T. R. Palfrey. 1995. Quantal response equilibria for normal form games. Games and Economic Behavior 10(1) 6-38.

[29] Naor, P. 1969. The regulation of queue size by levying tolls. Econometrica 37(1) 15-24.

[30] Rogers, B. W., T. R. Palfrey, C. F. Camerer. 2009. Heterogeneous quantal response equilibrium and cognitive hierarchies. Journal of Economic Theory 144(4) 1440-1467.

[31] Schweitzer, M. E., G. P. Cachon. 2000. Decision bias in the newsvendor problem with a known demand distribution: Experimental evidence. Management Science 46(3) 404-420.

[32] Stahl, D.O., P.W. Wilson. 1995. On players' models of other players: Theory and experimental evidence. Games and Economic Behavior 10(1) 218-254.

[33] Su, X. 2008. Bounded rationality in newsvendor models. Manufacturing Service Operations Management 10(4) 566-589.

[34] Su, X., F. Zhang. 2008. Strategic customer behavior, commitment, and supply chain performance. Management Science 54(10) 1759-1773. 


\section{Online Appendix A: Instructions}

Thank you for participating in this decision-making experiment. The instructions are simple; if you follow them carefully and make good decisions, you could earn a considerable amount of money, which will be paid to you in cash before you leave today. Different subjects may earn different amounts of cash. What you earn today depends partly on your decisions, partly on the decisions of others, and partly on chance.

The experiment will consist of 30 decision rounds. In each round, you will be randomly matched with another player in this room.

The decision making task is outlined as follows. Each player will take on the role of a retailer in a supply chain. Retailers order products, receive stock, and sell them on the market. In each decision round, you will make a single decision: how many units to order. The quantity of stock you receive may be different from your order and will depend on the orders chosen by you as well as the other player whom you are matched with. Your objective is to receive a quantity of stock that is as close as possible to the quantity demanded on the market (a fixed number of units). When the stock you receive is equal to the quantity demanded, you will earn the maximum possible profit. However, if you receive too much or too little stock, you will incur penalties that will be subtracted from your profit earnings. Therefore, your profit will depend on your order as well as the order of the other player whom you are matched with.

It is important that you do not look at the decisions of others, and that you do not talk, laugh or exclaim aloud during the experiment. You will be warned if you violate this rule the first time. If you violate this rule a second time, you will be asked to leave and you will not be paid. That is, your total earnings will be zero.

\section{Experimental Procedures}

The following steps will be repeated for every decision round that you participate in.

Step 1: Players submit orders. Each player chooses an integer number, ORDER, between 0 and 100. This is the number of units you would like to order. You will decide on your ORDER without seeing the decisions made by the other player.

Step 2: Players receive stock. There is a total of 90 units of stock available. The following procedure describes how these units will be divided between you and the player you are matched with. The computer calculates TOTAL ORDERS, which is the sum of the orders placed by you and the player you are matched with. This number is then revealed to both of you. If TOTAL ORDERS is less than or equal to 90 , the STOCK you receive is equal to the ORDER you placed. 
If TOTAL ORDERS is greater than 90, the STOCK you receive is calculated by the following formula: $\mathrm{STOCK}=($ ORDER / TOTAL ORDERS $) * 90$.

Let us consider following examples.

1. Suppose you placed an ORDER of 30 and TOTAL ORDERS is revealed to be 70 . Then, you will receive a STOCK of 30 .

2. Suppose you placed an order of 50 and TOTAL ORDERS is revealed to be 100 . Then, you will receive the following STOCK: STOCK $=50 / 100 * 90=45$.

Step 3: Players earn profit. The demand for each player is 50 units in every decision round. Your goal is to receive a STOCK as close as possible to 50 units. Depending on whether your STOCK is equal, less than, or greater than 50, you will receive point earnings as described below.

\section{Point Earnings}

If your STOCK is equal to 50, you earn the maximum possible 100 points in this round. If your STOCK is less than 50, you have too few units. For every unit that you are short, you incur a penalty of 2 points, which will be subtracted from the maximum possible of 100 points for this round. Therefore, your point earning this round will be: $100-2 *$ (50 - STOCK). If your STOCK is greater than or 50, you have too many units. For every excess unit that you have, you incur a penalty of 5 points, which will be subtracted from the maximum possible of 100 points for this round. Therefore, your point earning this round will be: $100-5 *$ (STOCK - 50).

\section{Final Dollar Payoff}

Your dollar earnings for the experiment will be determined as follows. First, we will add up your total point earnings from all 30 rounds. Then we will multiply your point earnings by 0.025 . That is, each point you earn is worth 0.025 RMB. This is the amount you will be paid when you leave the experiment. Note that the more points you earn, the more money you will receive. 


\section{Online Appendix B: Details on alternative explanations in Section 6}

Proposition 1. The Nash equilibrium is 100 when players are averse to negative profit, wastage, shortage, or inventory error, for both high-cost and low-cost conditions.

Proof: We provide the proof for the case of loss aversion. The other cases are similar. When players are loss averse, they face the utility function:

$$
u(x)=\left\{\begin{array}{l}
\pi(x), \pi(x) \geq 0 \\
\lambda \pi(x), \pi(x)<0
\end{array}\right.
$$

where $\lambda \geq 1$. Two properties of $\pi_{1}\left(x_{1}, x_{2}\right)$ are used in the proof: (i) $\pi_{1}\left(x_{1}, x_{2}\right)$ increases in $x_{1}$ when $x_{1} \leq \max \left\{1.25 * x_{2}, 50\right\}$; (ii) $\pi_{1}\left(x_{1}, x_{2}\right)$ decreases in $x_{1}$ when $x_{1} \geq \max \left\{1.25 * x_{2}, 50\right\}$. Next, we apply iterated strict dominance to our capacity allocation game.

Set $S^{0}=[0,100]$, and $x_{1}^{\prime}>x_{1}$.

If $x_{1}^{\prime}<50$, then $u_{1}\left(x_{1}^{\prime}, x_{2}\right)-u_{1}\left(x_{1}, x_{2}\right) \geq \pi_{1}\left(x_{1}^{\prime}, x_{2}\right)-\pi_{1}\left(x_{1}, x_{2}\right)>0$ for any $x_{2} \in S^{0}$. So any $x_{1}<50$ is strictly dominated. Thus $S^{1}=[50,100]$; this is the interval of best-response.

If $x_{1}^{\prime}<50 * D /(K-D)=50 * 1.25$, then $u_{1}\left(x_{1}^{\prime}, x_{2}\right)-u_{1}\left(x_{1}, x_{2}\right) \geq \pi_{1}\left(x_{1}^{\prime}, x_{2}\right)-\pi_{1}\left(x_{1}, x_{2}\right)>0$ for any $x_{2} \in S^{1}$. So any $x_{1}<50 * 1.25$ is strictly dominated. Thus $S^{1}=[50 * 1.25,100]$; this is the interval of best-response.

Let $S^{k}=\left\lfloor\underline{S^{k}}, \overline{S^{k}}\right\rfloor$, where $\underline{S^{k}}=\min \left\{1.25^{k-1} * 50,100\right\}$ and $\overline{S^{k}}=100$.

Finally, $\lim _{k \rightarrow \infty} \underline{S^{k}}=\overline{S^{k}}=100$, so the Nash equilibrium point is 100 .

The next result describes the equilibrium for the incomplete information game. Here, we consider a game where order quantities can be any real number in $[0,100]$ rather than integers only (The integrality constraint introduces notational complexities without changing the nature of the equilibrium).

Proposition 2. There exists two threshhold values $p_{1}^{*}=\frac{26 w}{26 w+27 s}$ and $p_{2}^{*}=\frac{40 w}{40 w+41 s}$ such that (1) if $p<p_{1}^{*}$, the Nash equilibrium is $(62.5,62.5)$; (2) if $p_{1}^{*} \leq p \leq p_{2}^{*}$, the Nash equilibrium is $(62.5,62.5)$ and $(100,100)$; and (3) if $p>p_{2}^{*}$, the Nash equilibrium is $(100,100)$.

Proof: Referring to the two properties of $\pi_{1}\left(x_{1}, x_{2}\right)$ in the previous proof, $\pi_{1}\left(x_{1}, x_{2}\right)$ is maximized at $x_{1}=\min \left\{\max \left\{1.25 * x_{2}, 50\right\}, 100\right\}$. Let $x_{1}^{*}\left(x_{2}\right)$ be the optimal value which maximizes $E \pi_{1}^{\prime}\left(x_{1}, x_{2}\right)=p \pi_{1}\left(x_{1}, x_{2}\right)+(1-p) \pi_{1}\left(x_{1}, 50\right)$. According to the two properties above, it holds that 


$$
x_{1}^{*}\left(x_{2}\right) \in \begin{cases}{[50,62.5]} & \text { when } x_{2} \leq 40 \\ (50,62.5] & \text { when } 40<x_{2} \leq 50 \\ {[62.5,78.13]} & \text { when } 50<x_{2}<62.5 \\ {\left[62.5, \min \left\{1.25 x_{2}, 100\right\}\right]} & \text { when } x_{2} \geq 62.5 .\end{cases}
$$

In our capacity allocation games, two players are symmetric, so the Nash equilibrium point should satisfy $x_{1}^{*}\left(x_{2}\right)=x_{2}$. From equation (8), we have that the Nash equilibrium point must be in the interval $\left[62.5, \min \left\{1.25 x_{2}, 100\right\}\right]$ if it exists.

Next, we discuss the extreme point when $x_{1} \in\left[62.5, \min \left\{1.25 x_{2}, 100\right\}\right]$ and $x_{2} \in\left[62.5, \min \left\{1.25 x_{1}, 100\right\}\right]$. In this case, $\pi_{1}\left(x_{1}, x_{2}\right)=100-s *\left(D-\frac{x_{1}}{x_{1}+x_{2}} K\right)$ and $\pi_{1}\left(x_{1}, 50\right)=100-w *\left(\frac{x_{1}}{x_{1}+50} K-D\right)$. It can be shown that $E \pi_{1}^{\prime}\left(x_{1}, x_{2}\right)=p \pi_{1}\left(x_{1}, x_{2}\right)+(1-p) \pi_{1}\left(x_{1}, 50\right)$ would be maximized at either the left bound 62.5 or the right bound $\min \left\{1.25 x_{2}, 100\right\}$, that is $x_{1}^{*}\left(x_{2}\right)=62.5$ or $\min \left\{1.25 x_{2}, 100\right\}$.

Finally, it can be shown that: (1) when $0 \leq p<\frac{26 w}{26 w+27 s}, x_{1}^{*}\left(x_{2}=62.5\right)=62.5$ and $x_{1}^{*}\left(x_{2}=\right.$ $100)=62.5$, so the Nash equilibrium is $(62.5,62.5) ;(2)$ when $\frac{26 w}{26 w+27 s} \leq p \leq \frac{40 w}{40 w+41 s}, x_{1}^{*}\left(x_{2}=\right.$ $62.5)=62.5$ and $x_{1}^{*}\left(x_{2}=100\right)=100$, so the Nash equilibrium is $(62.5,62.5)$ and $(100,100) ;(3)$ when $\frac{40 w}{40 w+41 s}<p \leq 1, x_{1}^{*}\left(x_{2}=62.5\right)=1.25 * 62.5$ and $x_{1}^{*}\left(x_{2}=100\right)=100$, then the Nash equilibrium is $(100,100)$.

In our capacity allocation games, $p_{1}^{*}=0.7939, p_{2}^{*}=0.7960$ for high-cost condition; and $p_{1}^{*}=$ $0.2781, p_{2}^{*}=0.2807$ for low-cost condition. 